\title{
Imaging Cold Molecules on a Chip
}

\author{
S. Marx, ${ }^{1}$ D. Adu Smith, ${ }^{1}$ M. J. Abel, ${ }^{1}$ T. Zehentbauer, ${ }^{1}$ G. Meijer, ${ }^{1,2}$ and G. Santambrogio ${ }^{1, *}$ \\ ${ }^{1}$ Fritz-Haber-Institut der Max-Planck-Gesellschaft, Faradayweg 4-6, 14195 Berlin, Germany \\ ${ }^{2}$ Radboud University of Nijmegen, Institute for Molecules and Materials, Heijendaalseweg 135, 6525 AJ Nijmegen, The Netherlands
}

(Received 16 October 2013; revised manuscript received 12 November 2013; published 12 December 2013)

\begin{abstract}
We present the integrated imaging of cold molecules in a microchip environment. The on-chip detection is based on resonance-enhanced multiphoton ionization, which is quantum state selective and generally applicable. We demonstrate and characterize time-resolved spatial imaging and subsequently use it to analyze the effect of a phase-space manipulation sequence aimed at compressing the velocity distribution of a molecular ensemble with a view to future high-resolution spectroscopic studies. The realization of such on-chip measurements adds the final fundamental component to the molecule chip, offering a new and promising route for investigating cold molecules.
\end{abstract}

DOI: 10.1103/PhysRevLett.111.243007

The idea of integrating various parts of a laboratory on a microchip is now over twenty years old and was motivated by a desire for simplification, reduction of costs, portability, speed of measurement, and ease of reproducibility. A reduced size allows for shorter transport times as well as for large field gradients, and therefore strong confinement forces created at low field strengths, employing only modest voltages or current densities. For physics, the atom chip [1] and ion chip [2] have been employed in fields as diverse as quantum computation [3], many-body nonequilibrium physics [4], and gravitational sensing [5]. For chemistry, the lab on a chip shrinks the pipettes, beakers, and test tubes of a modern lab onto a microchip-sized substrate [6], with applications from the international space station [7] to antiterrorism [8]. The molecule chip [9,10], however, is currently in its infancy, but promises a marriage between fundamental quantum physics and the richness of the chemical world. A particular advantage of using molecules instead of atoms on a chip is that they can be coupled to photons over a wider range of frequencies by their rotational and vibrational degrees of freedom. Moreover, for chemists, the molecule chip offers the prospect of extending the control of molecular concentrations and interactions to the level of single molecules with the accuracy in interaction energy enhanced to the $\mathrm{mK}$ level or beyond.

A major obstacle that has delayed the development of the molecule chip arises from this same richness of molecules' internal degrees of freedom. The complicated level structures of molecules result in a general lack of closed twolevel systems that are necessary for efficient laser cooling and detection using absorption or laser-induced fluorescence. In addition, in the presence of a physical structure such as a microchip, scattering or laser-induced fluorescence from surfaces adds noise to images, something which is critical when working with small samples. For these reasons, molecule detection has always been carried out tens of centimeters away from the chip. In recent years, we have
PACS numbers: $37.20 .+\mathrm{j}, 07.78 .+\mathrm{s}, 37.90 .+\mathrm{j}, 41.85 .-\mathrm{p}$

demonstrated that one can exploit the cooling provided by a supersonic expansion by loading the chip with cold molecules directly from a molecular beam [11]. The molecule chip has been used to trap, decelerate, and accelerate polar molecules [9], as well as manipulate both their rotational [12] and vibrational [13] states, using microwave and infrared radiation, respectively. This latter function can be used both as a spectroscopic tool and as a way to prepare a sample in a desired quantum state. Here we show on-chip molecule detection, adding the final fundamental component to the molecule chip. Our detection is based on resonance-enhanced multiphoton ionization (REMPI) [14], which we have already successfully employed in the past for off-chip detection $[12,13,15]$. REMPI is quantum state selective, can be saturated with a few $\mathrm{mJ} / \mathrm{mm}^{2}$ of laser light for most molecules, is intrinsically background-free, and is of general applicability. While in the simplest implementation of REMPI one would simply count the ions, we take the further step of using ion optics to create a time-resolved spatial image of the molecules.

We load the molecule chip directly from a molecular beam, which is generated by expanding a mixture of $20 \%{ }^{13} \mathrm{CO}$ in $\mathrm{Kr}$, cooled to $140 \mathrm{~K}$, through a pulsed solenoid valve operated at $10 \mathrm{~Hz}$. The expansion is skimmed to form a collimated beam with a mean velocity of $330 \mathrm{~m} / \mathrm{s}$ and intersected at right angles by a $206 \mathrm{~nm}$ laser pulse [16] exciting the molecules from the ground electronic state to the upper $\Lambda$-doublet component of the $a^{3} \Pi_{1}, v=0, J=1$ state. This metastable state has a lifetime of $2.6 \mathrm{~ms}$ [17] and consists of two low-field-seeking sublevels and a third sublevel with no significant Stark shift.

Our molecule chip is an array of gold microelectrodes on glass substrate [18]. By sinusoidally modulating the voltages applied to these electrodes, tubular microtraps that travel smoothly along the chip in the direction parallel to the molecular beam $z$ can be produced. The speed of 
the traveling traps is proportional to the frequency of the modulation. For example, a waveform of $2.75 \mathrm{MHz}$ frequency will move the traps at $330 \mathrm{~m} / \mathrm{s}$. Once the molecules are trapped they can be decelerated to a standstill by chirping down the frequency of the applied waveforms [9]. In all the experiments presented here, molecules are immediately decelerated to $138 \mathrm{~m} / \mathrm{s}$ upon arrival on the chip: this deceleration is sufficient to separate the trapped molecules from the background of untrapped molecules. The depth of the microtraps depends on the amplitude of the waveforms applied to the microelectrodes and on the acceleration of the traps (chirp rate of the waveforms), but not on the velocity of the traps (frequency of the waveforms).

For detection, the molecules are ionized using $(1+1)$ REMPI via the $b^{3} \Sigma^{+}, \quad v=0, \quad N=1$ state using $0.8 \mathrm{~mJ} / \mathrm{mm}^{2}$ of laser light at $283 \mathrm{~nm} \mathrm{[19]} \mathrm{that} \mathrm{propagates}$ parallel to the chip surface. For the simplest implementation of REMPI (i.e., no imaging) we ionize the molecules in the region of the microtraps after switching the traps off. All microelectrodes are switched to $0 \mathrm{~V}$ and, additionally, a rectangular ring electrode parallel to the chip surface, but offset above the chip by $4 \mathrm{~mm}$, is held at $-100 \mathrm{~V}$ [20]. This way the microelectrode array acts as an anode for the newly generated cations and the rectangular ring as a cathode. After flying through the cathode, the cations are then collected by a microchannel plate (MCP) detector.

The electric field homogeneity achievable above the microelectrode array, however, is not sufficient for spatial imaging. Therefore, we added an extra anode directly behind the microtraps region (Fig. 1), wider than the microtraps in the $x$ direction and $10 \mathrm{~mm}$ long in the $z$ direction. We hold this anode at $+80 \mathrm{~V}$. The molecules expand ballistically while they fly above the anode after being ejected from the microtraps. Hence this electrode is recessed by $2 \mathrm{~mm}$ under the plane of the traps to leave enough space for the expansion. As a cathode we use the same rectangular ring as for the nonimaging detection. For imaging, the cathode is held at $-130 \mathrm{~V}$. Together the anode and cathode reduce the inhomogeneity of the electric field and therefore any associated imaging aberrations. The ions generated by REMPI are imaged using ion optics onto a MCP detector with phosphor screen situated $40 \mathrm{~cm}$ above the chip surface and recorded using a fast CCD camera.

The ion optics for our experiment are a standard set of three asymmetric ion lenses located $26 \mathrm{~mm}$ from the chip surface, spaced by $10 \mathrm{~mm}$ and all held at $-400 \mathrm{~V}$. The openings in the centers of the ion lenses of 22 by $72 \mathrm{~mm}$ provide magnification factors at the MCP of 2 and 58 , in the direction of the tubular trap axis and the direction of the trap movement, respectively. We chose this asymmetric magnification because of the large aspect ratio of the tubular microtraps (200). Four images are shown in Fig. 2. They show molecules ejected from the microtraps toward the detection region, after a ballistic expansion of 9 ,

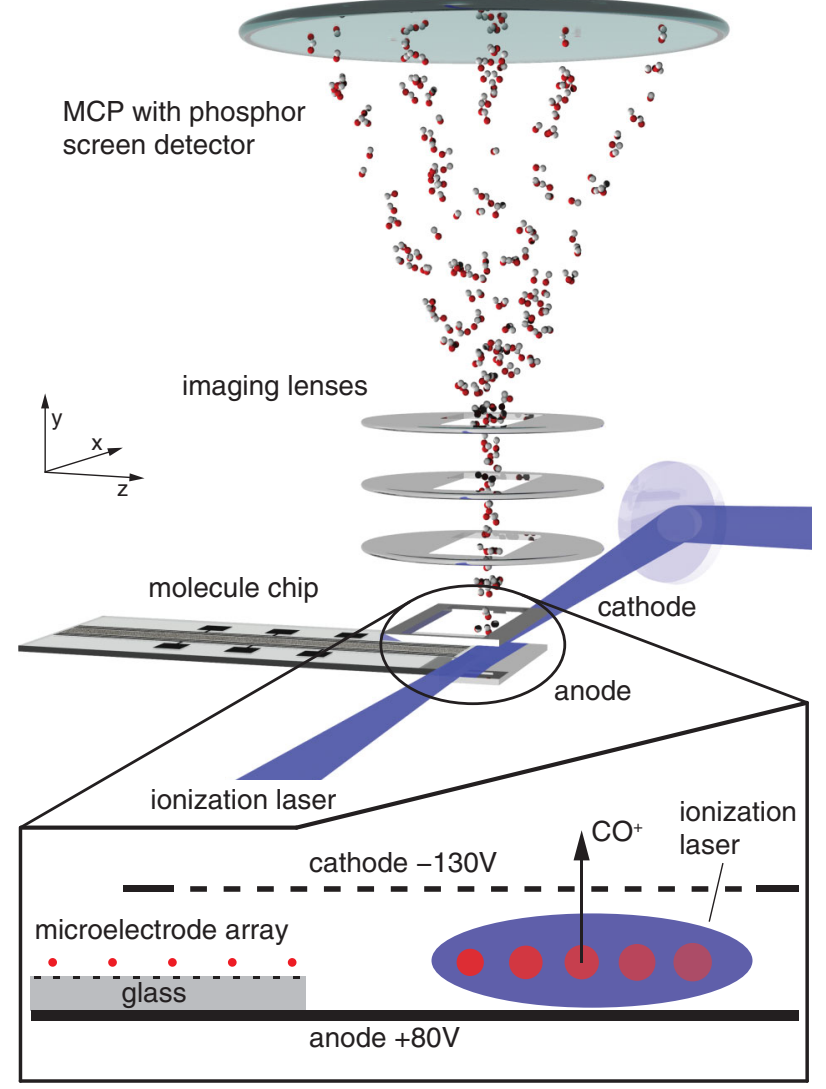

FIG. 1. Metastable CO molecules on the molecule chip are ionized via REMPI using a pulsed $283 \mathrm{~nm}$ laser. The detection region is composed of two electrodes: an anode and a cathode. The cathode is a rectangular ring. Together the two electrodes protect the cations from stray fields in the region of the chip. Ion optics are then used to form an image on a MCP detector backed by a phosphor screen. A CCD camera (not shown) is used to record the image of the molecules from the phosphor screen. A sketch of the detection region is shown in the inset, not to scale. Two points in time are shown: on the left, when the molecules are still trapped above the microelectrode array; on the right, after ballistic flight, upon reaching the axis of the imaging lenses.

15,19 , and $22 \mu$ s. One can clearly distinguish the single traps and follow the ballistic expansion of the molecular clouds. At a still relatively short time of flight of $22 \mu \mathrm{s}$, the resolution of the individual microtraps is almost completely washed out. This highlights the importance of on-chip detection for spatial imaging. The REMPI laser fires when the molecules are on the axis of the imaging lenses. With the distance between release and detection being fixed, the time for which molecules are in ballistic flight is given by the center-of-mass velocity of the molecular ensemble. We control this velocity by choosing the speed of the microtraps at the point at which the molecules are released; i.e., a lower microtraps velocity gives a longer time for ballistic flight. For the ballistic expansion images shown in Fig. 2, the molecules were released when traveling uniformly at 336, 207, 162, and $138 \mathrm{~m} / \mathrm{s}$, respectively, 


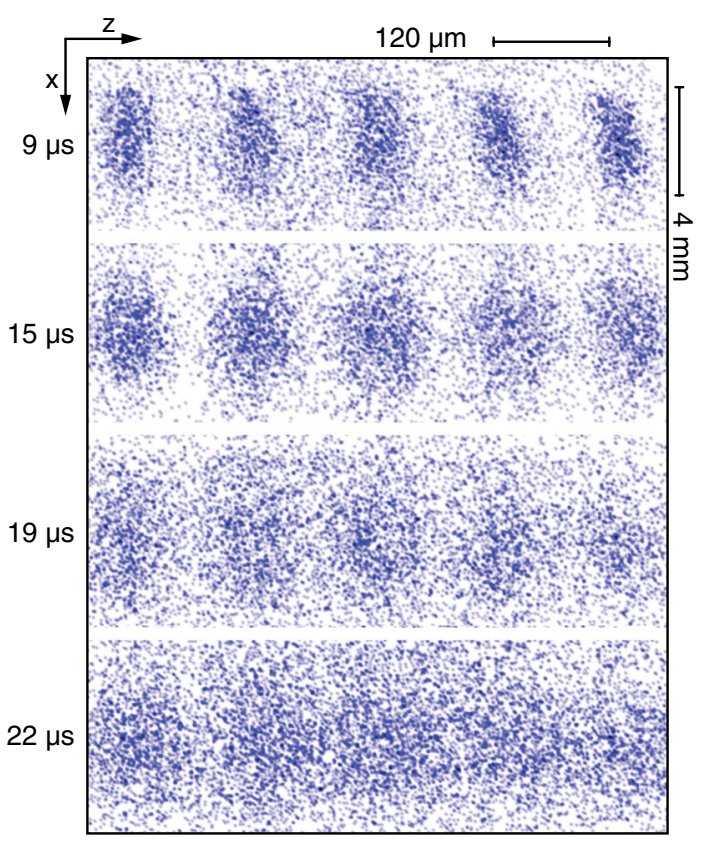

FIG. 2 (color online). Two-dimensional spatial imaging of CO molecules. Molecules are imaged for different ballistic expansion times. The vertical direction is along the length of the microtraps, $x$. The extreme aspect ratio of the microtraps, $x: z=200: 1$, is reduced to approximately $7: 1$ by the asymmetric magnification factors of the lenses. Some ion-optics aberrations can be observed on the right-hand side of the image, in particular at shorter times. The experimental procedure runs at a rate of $10 \mathrm{~Hz}$ and the images are the sum of approximately $10^{5}$ experimental cycles. We record approximately 1-2 counts/s. The resolution of the imaging system in the $z$ direction is $0.78 \mathrm{pixel} / \mu \mathrm{m}$.

for the expansion times of $9,15,19$, and $22 \mu$ s to cover the 3 -mm distance between the point of release from the microtraps and the axis of the imaging lenses in the detection region. Each microtrap releases its molecules upon reaching the end of the microelectrode array; i.e., the clouds are released sequentially, each time at the same position. [21] The expansion time is given for the central cloud of each image, whereas the clouds at either side expand for slightly shorter or longer times. With the signal-to-noise ratio of the data presented here, the small differences in the cloud widths within the same image, due to this difference in expansion time, are masked by the experimental noise (see also Fig. 3). The purpose of recording measurements at different times is to follow the evolution of the system. This, of course, is only meaningful if the initial conditions are the same for each measurement. We therefore made sure that the molecules experienced the same trap depth and shape for every measurement. We achieved different microtraps velocities at the point of release by applying a fixed acceleration of $10^{6} \mathrm{~m} / \mathrm{s}^{2}$ for different time durations. A waveform amplitude of $160 \mathrm{~V}$ (peak-to-peak) applied to the microelectrodes creates $28 \mathrm{mK}$ deep traps for $\mathrm{CO}$ molecules in low-field-seeking components of the $a^{3} \Pi_{1}$, $v=0, J=1$ state, under an acceleration of $10^{6} \mathrm{~m} / \mathrm{s}^{2}$.
Upon reaching the desired final velocity, the motion is made uniform and this increases the trap depth to $57 \mathrm{mK}$ while the trap diameter increases from 4 to $20 \mu \mathrm{m}$.

The dynamics of the molecules along the 4-mm length of the microtraps ( $x$ direction) is negligible for the experiments presented here because the molecules almost never experience a force in that direction during the relatively short time they spend on the chip. We therefore integrate the signal along the $x$ direction (vertical axis of the images) and concentrate on the perpendicular direction. The results are shown in Fig. 3(a) together with the results of numerical trajectory simulations. Experiments and simulations show good agreement, demonstrating that both the ballistic expansion and the ion imaging process are not significantly hindered by stray electric fields.

We have a moleculardensity of $10^{7} / \mathrm{cm}^{3}$ in the traps, which corresponds to about five molecules per microtrap. The sensitivity of our REMPI detection allows us to work under such conditions. Indeed, analysis of the trajectory simulations shows that this trapped ensemble of molecules does not have a perfect thermal distribution. However, a comparison of the molecular velocities to a Maxwell-Boltzmann distribution remains helpful for understanding the orders of magnitude

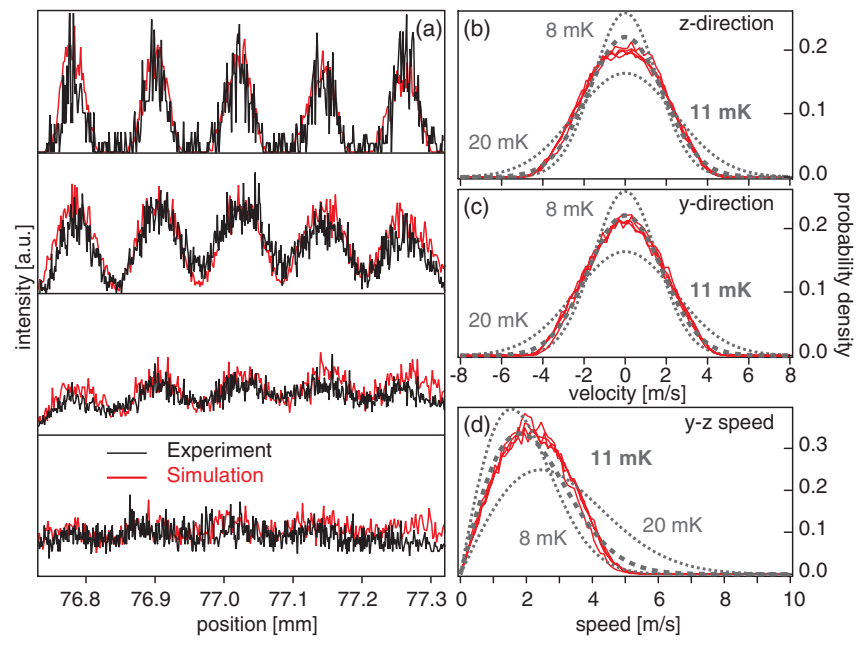

FIG. 3. (a) Integrated signals measured under the same conditions as in Fig. 2, together with trajectory simulations. The experimental signals are each scaled to the (unscaled) simulations, which, for each expansion time, were run with the same number of input molecules. (b) and (c) Velocity distributions in the $z$ and $y$ directions from trajectory simulations (four almost overlapping solid red lines). Results are shown for all four sets of data in (a). For comparison, calculated Maxwell-Boltzmann distributions are plotted for 8 and $20 \mathrm{mK}$ (dotted lines) and $11 \mathrm{mK}$ (dashed line), which is the best numerical fit. (d) Speed distributions in the $y-z$ plane from trajectory simulations (solid red lines) and corresponding Maxwell-Boltzmann distributions. While the simulated velocity distributions appear well approximated by thermal distributions, the deviation of the speed distribution indicates that there is some correlation between the velocities in the $y$ and $z$ directions. 
of the observed phenomena. In Figs. 3(b)-(d), the calculated velocity distributions in the $z$ and $y$ directions are shown together with the speed distribution in the $y-z$ plane. All velocities are relative to the mean velocity. MaxwellBoltzmann distributions are also plotted for comparison, with the molecular clouds having a best-fit temperature of $11 \mathrm{mK}$.

Potential developments for future molecule chips include the integration of, for example, optical traps [22], as well as cooling techniques to reach ultracold temperatures and the implementation of high-resolution spectroscopy [23]. If the molecule chip were to be used as a molecular source for high-resolution spectroscopy, the molecules would need to be ejected at low velocity from the chip with a narrow velocity distribution so that the packet of molecules remains together for the longest possible time [24]. Indeed this is one of the main goals of cold-molecule research. As a demonstration of the new on-chip detection system, we use it to analyze the effect of a phase-space manipulation sequence to compress the velocity distribution without losing molecules. With CO molecules in deep microtraps, we slowly reduce the trap depth while guiding the molecules at constant velocity across the chip surface. Analysis of trajectory simulation results shows that adiabatically changing the trap depth from 72 to $13 \mathrm{mK}$ in $188 \mu \mathrm{s}$ (slow compared to the trap frequencies of hundreds of $\mathrm{kHz}$ ) leads to a reduction of the best-fit temperature of the molecular ensemble to roughly a third of its initial value. Integrated imaging signals along with corresponding trajectory simulations are shown in Fig. 4. The experimental data show a clearly narrower spatial structure in the case of the manipulated molecules, indicating that, over the expansion time of $15 \mu \mathrm{s}$, the manipulated molecules have expanded less. The in-trap spatial expansion that results from the phase-space manipulation can be neglected for most practical purposes because the size of the molecular clouds after release is entirely dominated by the expansion velocity even at very short times, as can be seen in Fig. 4. Crucially, we lose less than $5 \%$ of the molecules during the manipulation process. It is also possible to use our imaging setup for velocity map imaging [25]. However, with the present ionization scheme, ion recoil energies are of the order of tens of $\mathrm{mK}$, i.e., comparable with the trap depths, which makes this method imprecise for the analysis of the velocity distributions inside the microtraps.

The chief advantage of on-chip detection is the ability to probe the system at short evolution times. This allows for the detection of short-lived quantum states. Moreover, it maximizes spatial resolution and increases the signal-tonoise ratio, as it avoids the ballistic expansion of the molecules on their way to an external detector. Here we have presented an on-chip detector that fulfills all these requirements. One of its main advantages is its general applicability, through the use of the REMPI process, making it not only quantum state selective, but also applicable to virtually every molecule. This development of the molecule chip
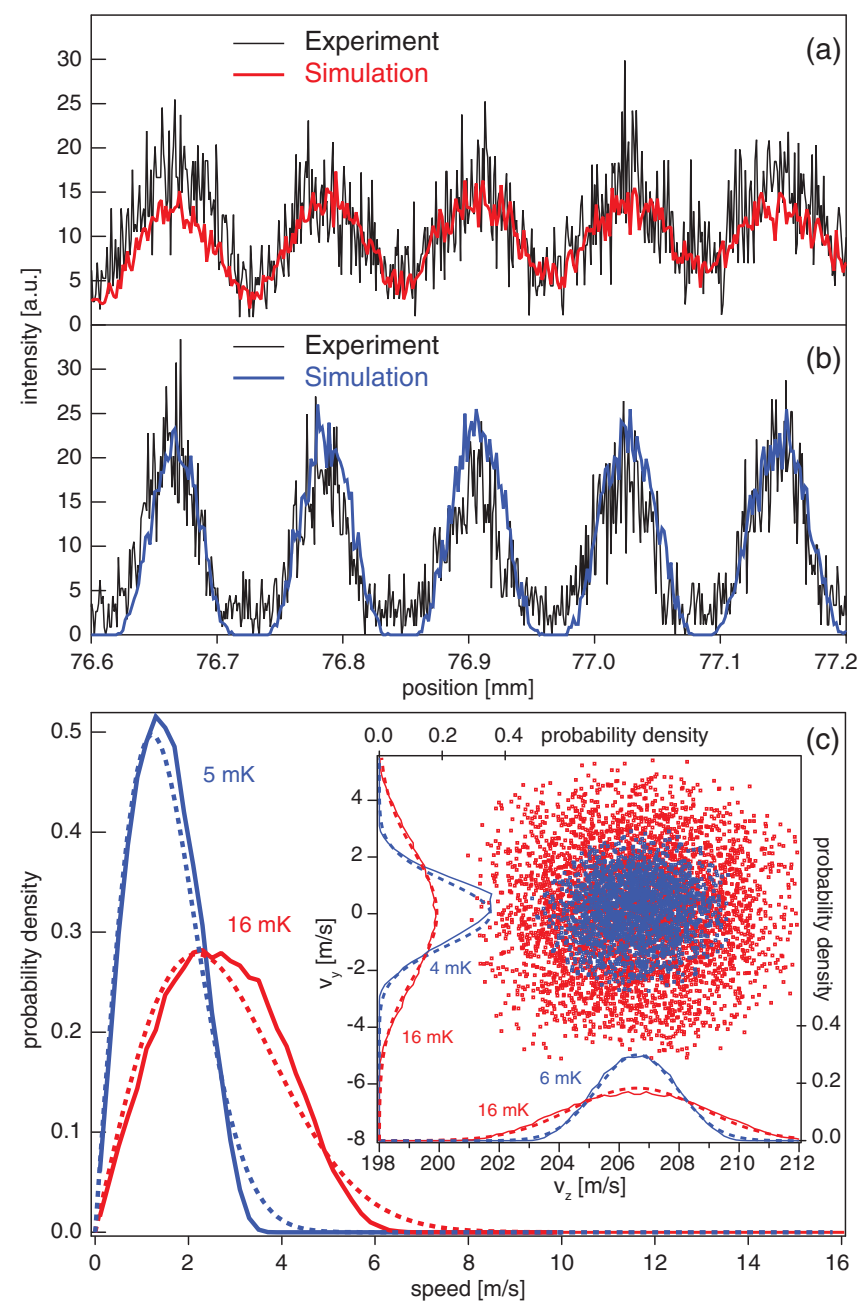

FIG. 4. Integrated signals for molecules guided in traps with constant depth (a) and decreasing depth (b), together with results from trajectory simulations. There is no rescaling between (a) and (b). (c) Speed distribution in the $y-z$ plane for the same conditions as in (a) and (b). The inset in (c) shows the two-dimensional velocity distribution with projections in both directions. The distributions in the $y$ and $z$ directions after phase-space manipulation are different, showing that the phase-space manipulation does not act isotropically. Results from trajectory simulations are shown with solid lines, while Maxwell-Boltzmann distributions for the best-fit temperatures are shown as dashed lines. Simulations show a number loss of approximately $5 \%$ during the phase-space manipulation.

to a complete environment that can trap, manipulate, and detect molecules is significant progress towards a tool for the control of single and small samples of molecules for investigating a wide range of quantum phenomena and molecular processes.

We gratefully acknowledge the work of the electronic laboratory of the Fritz Haber Institute, in particular Georg Heyne and Viktor Platschkowski, as well as fruitful discussions with Samuel A. Meek. This work has been funded by the European Community's Seventh Frame- 
work Program FP7/2007-2013 under Grant Agreement No. 216774 and ERC-2009-AdG under Grant Agreement No. 247142-MolChip.

*gabriele.santambrogio@fhi-berlin.mpg.de

[1] Atom chips, edited by J. Reichel and V. Vuletic (WileyVCH, New York, 2010).

[2] D. Stick, W. K. Hensinger, S. Olmschenk, M. J. Madsen, K. Schwab, and C. Monroe, Nat. Phys. 2, 36 (2006).

[3] C. Ospelkaus, U. Warring, Y. Colombe, K. R. Brown, J. M. Amini, D. Leibfried, and D. J. Wineland, Nature (London) 476, 181 (2011).

[4] M. Gring, M. Kuhnert, T. Langen, T. Kitagawa, B. Rauer, M. Schreitl, I. Mazets, D. Adu Smith, E. Demler, and J. Schmiedmayer, Science 337, 1318 (2012).

[5] T. van Zoest et al., Science 328, 1540 (2010).

[6] R. Daw and J. Finkelstein, Nature (London) 442, 367 (2006).

[7] H. C. Morris, M. Damon, J. Maule, L. A. Monaco, and N. Wainwright, Astrobiology 12, 830 (2012).

[8] T. Frisk, D. Ronnholm, W. van der Wijngaart, and G. Stemme, Lab Chip 6, 1504 (2006).

[9] S. A. Meek, H. Conrad, and G. Meijer, Science 324, 1699 (2009).

[10] Martin Zeppenfeld, Barbara G. U. Englert, Rosa Glockner, Alexander Prehn, Manuel Mielenz, Christian Sommer, Laurens D. van Buuren, Michael Motsch, and Gerhard Rempe, Nature (London) 491, 570 (2012).

[11] S. A. Meek, H. L. Bethlem, H. Conrad, and G. Meijer, Phys. Rev. Lett. 100, 153003 (2008).

[12] G. Santambrogio, S. A Meek, M. J. Abel, L. M. Duffy, and G. Meijer, ChemPhysChem 12, 1799 (2011).
[13] M. J. Abel, S. Marx, G. Meijer, and G. Santambrogio, Mol. Phys. 110, 1829 (2012).

[14] M. N. R. Ashfold and J. D. Howe, Annu. Rev. Phys. Chem. 45, 57 (1994).

[15] S. A. Meek, G. Santambrogio, B. G. Sartakov, H. Conrad, and G. Meijer, Phys. Rev. A 83, 033413 (2011).

[16] L. Velarde, D. P. Engelhart, D. Matsiev, J. LaRue, D. J. Auerbach, and A. M. Wodtke, Rev. Sci. Instrum. 81, 063106 (2010).

[17] J. J. Gilijamse, S. Hoekstra, S. A. Meek, M. Metsälä S. Y. T. van de Meerakker, G. Meijer, and G. C. Groenenboom, J. Chem. Phys. 127, 221102 (2007).

[18] S. A Meek, H. Conrad, and G. Meijer, New J. Phys. 11, 055024 (2009).

[19] Light at $283 \mathrm{~nm}$ is obtained by frequency doubling the output of a NarrowScan from the Radiant Dyes Laser, pumped by the second harmonic of a Nd:YAG laser.

[20] The exact voltage applied to the rectangular ring electrode for nonimaging detection is not critical.

[21] Numerical simulations show that each trap rapidly opens out upon arrival at the end of the microelectrode array. This happens within hundreds of nanoseconds, i.e., instantaneously for the molecules. The fields disappear with a monotonic decrease in the electric field strength gradient.

[22] M. Padgett and R. Di Leonardo, Lab Chip 11, 1196 (2011).

[23] L. Carr, D. DeMille, R. Krems, and J. Ye, New J. Phys. 11, 055049 (2009).

[24] M. Quintero-Pérez, P. Jansen, T. E. Wall, J. E. van den Berg, S. Hoekstra, and H. L. Bethlem, Phys. Rev. Lett. 110, 133003 (2013).

[25] A. T. J. B. Eppink and D. H. Parker, Rev. Sci. Instrum. 68, 3477 (1997). 